\title{
Fine Structure Analysis of Ramie Fibres by Small Angle X-Ray Scattering Technique
}

\author{
T. MisRa, J. Shukla, Md. N. Khan, ${ }^{*}$ \\ D. K. BISOYI,** and T. PATEL \\ Regional Engineering College, Rourkela-769008, Orissa, India \\ * Department of Phvsics, IGIT Saranga, Talcher, Orissa, India \\ ** Department of Physics, Gandhi Mahavidyalaya, Rourkela, Orissa, India
}

(Received May 6, 1997)

\begin{abstract}
Small Angle X-ray Scattering due to degummed ramie fibres were recorded using a Compact Kratky Camera, having counter device. The fibres were taken both in natural state and alkali treated state at room temperature. Various macromolecular parameters, such as $D$ the average periodicity transverse to layer, $S / V$, the specific inner surface, $E$, the width of transition layer, $T_{1}, T_{2}, T_{\mathrm{r}}$, transversal lengths $l_{\mathrm{c}}$ length of coherence etc. were calculated by using the correlation functions of the smeared out scattering curve of the different samples. Increase in the value of $D$ with concentration of sodium hydroxide solution may be due to swelling of macromolecules present in the fibre. The calculation of various parameters were done by applying theories of Ruland ${ }^{14}$ and Vonk. ${ }^{7}$

KEY WORDS Small Angle / Small Angle X-Ray Scattering / Non-Ideal Two Phase System / Correlation Functions / Periodicity Transverse to The Layer / Width of Transition Layer /
\end{abstract}

Ramie is a natural cellulosic fibre. Being the longest and strongest fibre of vegetable origin, it has suitable textile uses. ${ }^{1}$ It is often blended with cotton to improve its textile properties. ${ }^{2}$ As Ramie is mostly cellulosic, it comes under the macromolecular system. We present here the SAXS studies on ramie fibres to compare the macromolecular structure of natural fibre with fibres treated in sodium hydroxide solution at different concentration.

The work of Misra et al. ${ }^{3,4}$ on natural cellulosic fibre like cotton, sisal etc. shows that natural cellulosic macromolecules possess non-ideal two phase structure. Ramie being a natural polymer, exists in a semicrystalline state. On analysing the scattering data of the samples it is revealed that, ramie fibre belongs to non-ideal two phase system.

According to Porod ${ }^{5.6}$ for an ideal two phase densely packed system, with sharply defined phase boundaries, the product $\widetilde{I}(\theta) \theta^{3}$ comes out to be constant at the tail end of small angle X-ray scattering (SAXS) curve, here $\widetilde{I}(\theta)$ is smeared out intensities and $\theta$ is half the scattering angle. It has been shown by Vonk ${ }^{7}$ that above product doesn't remain constant and decreases with $\theta$, as electron density changes continuously over a certain region between the two phases (i.e., matter and void) known as width of transition layer $\mathrm{E}$.

\section{EXPERIMENTAL}

Ramie fibre was collected from Faculty of Textile Science and Technology, Shinshu University, Tokida, Japan. It is silky white in colour and lusturous in appearance: The fibre is least affected by moisture and highly resistant to bacteria and fungi. It is also very durable and can easily be bleached and dyed. Ramie can most satisfactorily be used for same purpose as cotton, flax and hemp. Natural Ramie fibre contains some gummy matter which is a heterogeneous mixture of carbohydrates of a complex chemical nature. ${ }^{8}$ So the sample was degummed by the process reported by Roy. ${ }^{9}$ As a result of this, gum was removed and the sample attains a hohlraum character. ${ }^{10.11} \mathrm{~A}$ part of the sample was soaked in sodium hydroxide solution at concentrations $\mathrm{pH} 11$ and $\mathrm{pH} 12$ for $48 \mathrm{~h}$ at room temperature and then it was washed thoroughly with distilled water and dried.

SAXS intensity data for both untreated (Hence forth called air-dried sample or sample-I) and treated samples (with sodium hydroxide $\mathrm{pH} 11$ and $\mathrm{pH} 12$ called sampleII and sample-III, respectively) were collected using a compact Kratky Camera having counter device. The source of energy was a Phillips P.W 1729 X-ray generator. The entrance slit was of $80 \mu \mathrm{m}$ width and Copper target was operated at $40 \mathrm{kV}$ and $30 \mathrm{~mA} .{ }^{12}$ Counter slit had a width of $200 \mu \mathrm{m}$. Filtered $\mathrm{Cu}-K_{\alpha}$ radiation $(\hat{\lambda}=1.54 \AA)$ was used to irradiate the sample. Sample to counter distance was maintained at $20 \mathrm{~cm}$. To avoid parasitic scattering by air present between sample and detector the irradiation was carried out under a vacuum of approximately $1 / 2 \mathrm{hPa}$, as reported by Hendricks. ${ }^{13}$ The room temperature was kept at $22.5 \pm 0.5^{\circ} \mathrm{C}$ during the $\mathrm{X}$-ray intensity data collection. X-Ray intensity data was taken both for the sample in the capillary tube and also for the blank capillary tube. To plot the smeared out intensity curve (Figure 1), the blank capillary data was subtracted from the observed scattering data. To test the isotropic property of the sample intensity data (of sample-I) in its natural orientation (i.e., in vertical direction) was also recorded (Figure 2). Constant background intensity has been subtracted from observed data according to Konard and Zachmann ${ }^{22}$ for the purpose of evaluation of different parameters of the samples.

\section{THEORY}

Theories of Vonk ${ }^{7}$ and Ruland ${ }^{14}$ have been applied here to calculate various macromolecular parameters, treating the sample as a non-ideal two phase system having isotropic structure. An important parameter in 
characterisation of structure is given by

$$
R=\frac{\left\langle|\operatorname{grad} \eta|^{2}\right\rangle}{\left\langle\eta^{2}\right\rangle}=4 \pi \frac{\int_{0}^{\infty} s^{4} I(s) \mathrm{d} s}{\int_{0}^{\infty} s^{2} I(s) \mathrm{d} s}=6 \pi^{2} \frac{\int_{0}^{\infty} s^{3} \boldsymbol{I}(s) \mathrm{d} s}{\int_{0}^{\infty} s \boldsymbol{I}(s) \mathrm{d} s}
$$

Where $I(s)$ and $I(s)$ are desmeared and smeared out intensities respectively in arbitrary units, $\eta$ is deviation of electron density from its mean value, $s$ is a vector in a reciprocal (Fourier) space. In an ideal two phase system, the gradient at phase boundary is infinity and $R$ also goes to infinity. The case where the minimum value of $R=4 \pi^{2} s_{0}{ }^{2}$ corresponds to the intensity function $I(s)$ consisting of a single peak at $s=s_{0}$. Such an intensity function is obtained from a randomised layer structure. Here electron density perpendicular to layers fluctuates according to a sine function.

Further $R$ can be expressed in terms of intensity function $I(x)$ by Misra et al. ${ }^{3,16}$

$$
R=3 / 2(2 \pi / \lambda a)^{2} \int_{0}^{\infty} x^{3} I(x) \mathrm{d} x / \int_{0}^{\infty} x \boldsymbol{I}(x) \mathrm{d} x
$$

where $x$ is a position co-ordinate of the scattered intensity from the center of primary beam, $a$, distance of sample from the counter slit and $s=2 \theta / \lambda=x / \lambda a, 2 \theta$ is the scattering angle. Small, positive and finite value of $R$ suggests that corrugation at the phase boundary exists and electron density changes from phase I to phase II over a transition layer $E$ and in consequence Porod's law, fails.

The width of transition layer can be obtained from the relation derived by Vonk $^{7}$ is

$$
E=(-4 / R)(\mathrm{d} C(r) / \mathrm{d} r)_{r=E}
$$

$C(r)$ is the 3-dimensional correlation function of a sample normalised to unity at the origin in real space. The function was derived by Mering and Tchoubar $^{15}$ and expressed in terms of ' $x$ ' by Misra et $a l .{ }^{16}$ as

$$
C(r)=\int_{0}^{\infty} x \boldsymbol{I}(x) J_{0}(2 \pi r x / \lambda a) \mathrm{d} x / \int_{0}^{\infty} x \boldsymbol{I}(x) \mathrm{d} x
$$

$J_{0}$ is the Bessel function of zero order of first kind. According to Vonk $^{7}$ for an isotropic sample

$$
R=-3\left(\mathrm{~d}^{2} C(r) / \mathrm{d} r^{2}\right)_{r=0}
$$

One-dimensional correlation function $C_{1}(y)$ given by Kortleve and Vonk $^{17}$ for layered structure in terms of $x^{16}$ is expressed as

$$
C_{1}(y)=\int_{0}^{\infty} x \boldsymbol{I}(x)\left[J_{0}(z)-z J_{1}(z)\right] \mathrm{d} x / \int_{0}^{\infty} x \boldsymbol{I}(x) \mathrm{d} x
$$

Where $z=2 \pi x y / \lambda a$ and $J_{1}$ is the Bessel function of first order and of first kind. According to $\mathrm{Vonk}^{7}$ the position of the first subsidiary maximum in the one dimensional correlation function $C_{1}(y)$, gives the value of average periodicity $D$ transverse to the layers.

$$
\text { The specific inner surface } S / V=2 / D
$$

is defined as the phase boundary per unit volume of the 1008

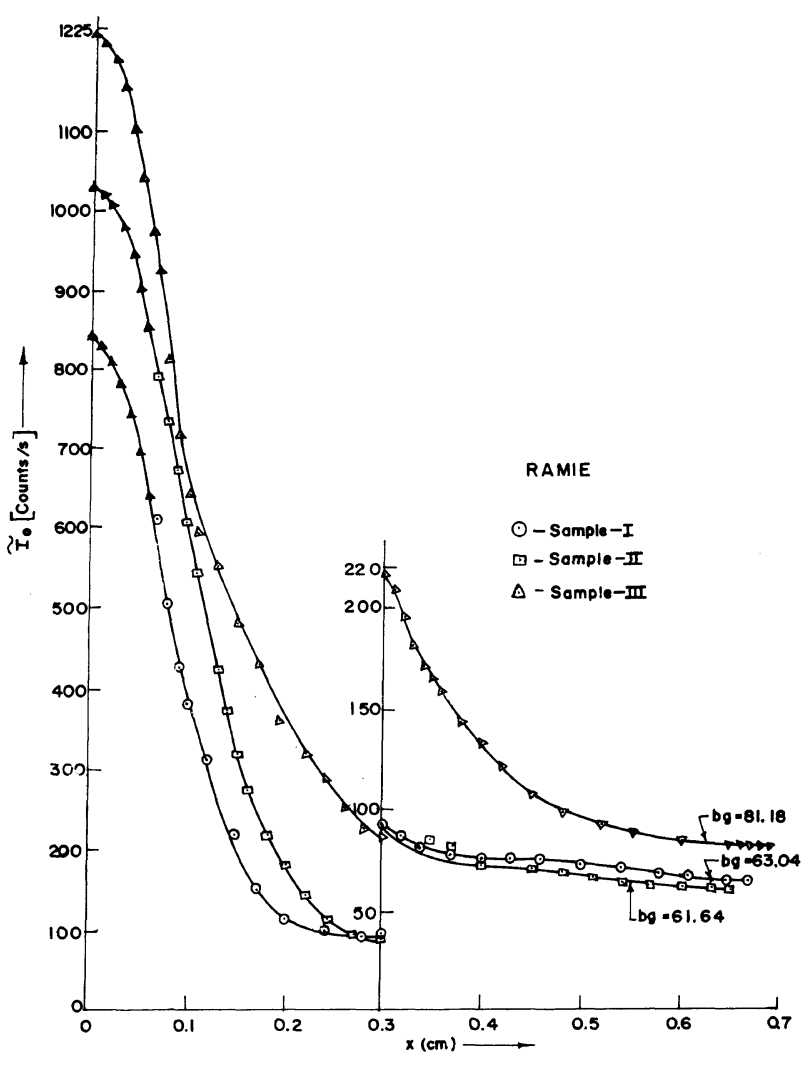

Figure 1. Smeared out scattering curves.

dispersed phase.

The relation

$$
\left\langle\eta^{2}\right\rangle /\langle\Delta \eta\rangle^{2}=\phi_{1} \phi_{2}-(E S / 6 V)
$$

was given by Vonk ${ }^{7}$ where $\phi_{1}, \phi_{2}$ are the volume fraction of matter and void regions respectively and relation $\phi_{1}+\phi_{2}=1$ can be used to determine the individual values of $\phi_{1}$ and $\phi_{2}$.

As defined by Mittelbach and Porod ${ }^{18}$ for a two phase system transversal lengths of matter and void phases for a 3 dimensional system are given by the relation.

$$
\begin{array}{ll} 
& T_{1}=4 \phi_{1}(V / S), \quad T_{2}=4 \phi_{2}(V / S) \\
\text { and } \quad 1 / T_{\mathrm{r}}=1 / T_{1}+1 / T_{2}
\end{array}
$$

Where $\bar{l}_{r}$ is the range of inhomogeneity.

According to Ruland ${ }^{19}$ and using Porod's law, it is found that

$$
\begin{aligned}
& 1 / d_{\mathrm{r}}=1 / d_{1}+1 / d_{2} \\
& d_{\mathrm{r}}=I_{\mathrm{r}} / 2, \quad d_{1}=\bar{l}_{1} / 2, \quad d_{2}=\bar{l}_{2} / 2 \\
& l_{\mathrm{c}}=2 \int_{0}^{\infty} C(r) \mathrm{d} r
\end{aligned}
$$

and

Where $d_{1}$ and $d_{2}$, numbers related to average thickness in the matter and void regions respectively, $l_{\mathrm{c}}$ is the length of coherence, which is the average value of length of cords drawn in all directions. A second method to determine width of transition layer $E$ given by Ruland ${ }^{14}$ for a non-ideal two phase system expressed in terms of $x$ is given by

$$
I(x \rightarrow \infty) \cdot x=\pi c / 2 \cdot(\lambda a)^{3} \cdot x^{-2}-\pi^{3} c / 3 \cdot(\lambda a) E^{2}
$$

From the above equation the plot of $I(x) \cdot x$ versus $x^{-2}$ 


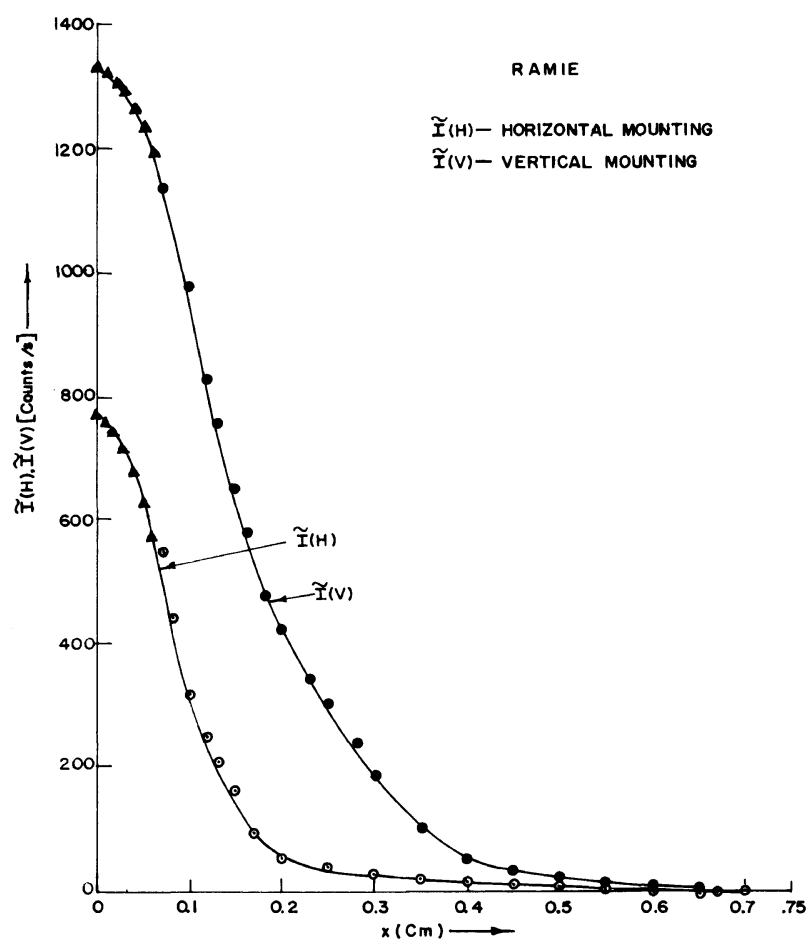

Figure 2. Smeared out scattering curve.
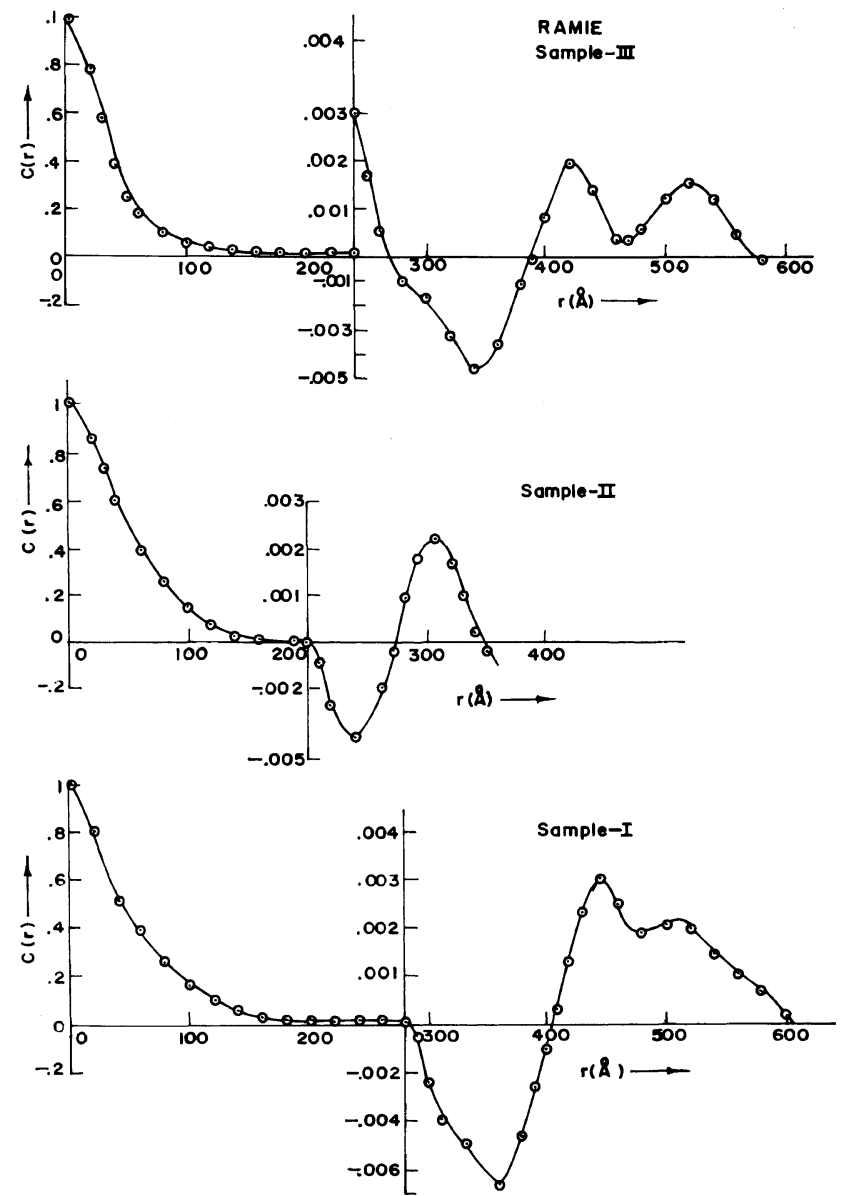

Figure 3. Curves of the three-dimensional correlation function $C(r)$ against ' $r$ '.

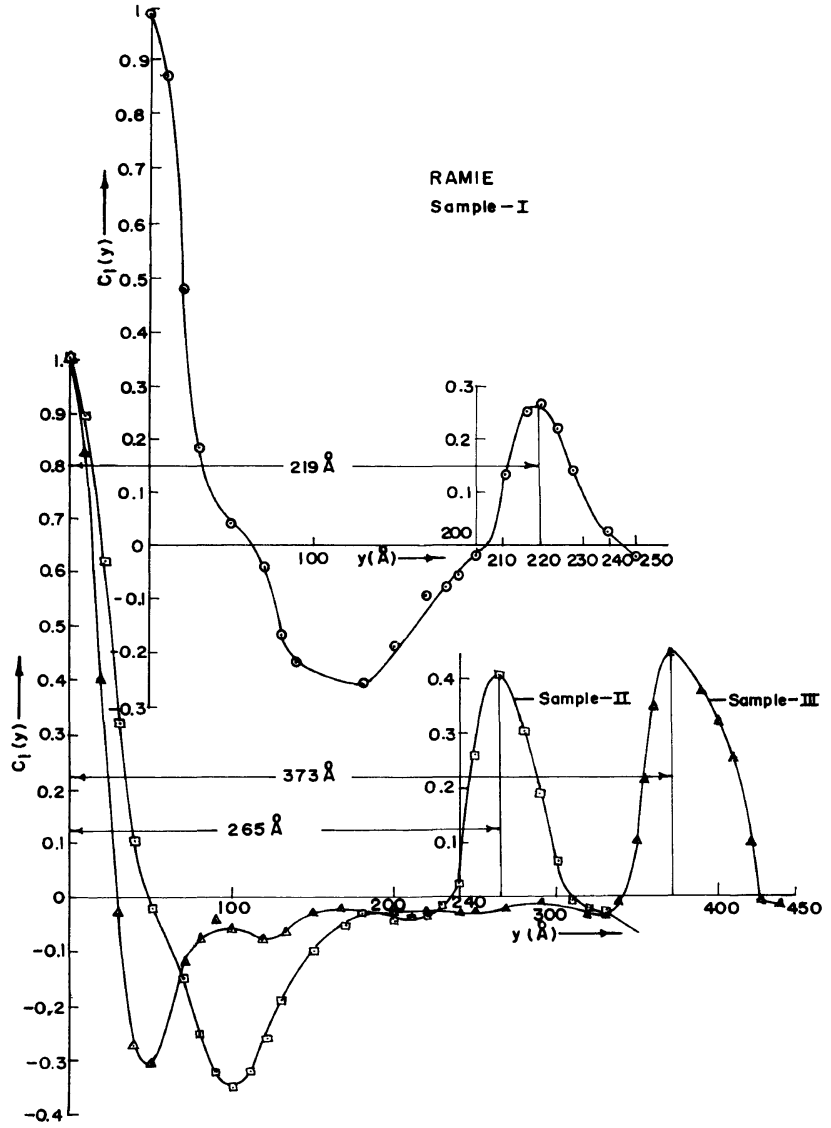

Figure 4. Curves of the one-dimensional correlation function $C(y)$ against ' $y$ '.

at the tail end of the SAXS pattern is known as Ruland Plot. Equation 14 reduces to Porods law if $E=0$, for an ideal two phase system.

\section{RESULTS AND DISCUSSION}

Intensity values $\boldsymbol{I}(x)$ for five points near the origin were fitted to Gauss curve ${ }^{20}$

$$
I(x \rightarrow 0)=P \exp \left(-q x^{2}\right)
$$

by least square technique. The values of $P$ for sample, I, II, and III were obtained as $838.174,1029.76,1217.57$, respectively and corresponding values of $q$ as $89.59,53.47$, and 62.075, respectively. Using $P$ and $q$ the scattering pattern was extrapolated to $x \rightarrow 0$ angle.

The extrapolated points for all samples were indicated by symbol $\boldsymbol{\Delta}$ in Figures 1 and 2. Extrapolating to zero angle has very little effect on relevant part of the correlation functions. Position and height of first subsidiary maximum of one-dimensional correlation function is not much affected by extrapolation. ${ }^{16}$ Values of $R$ were determined using eq 2 are found out to be $3.523 \times 10^{-3}$ $\AA^{-2}, 2.329 \times 10^{-3} \AA^{-2}, 3.771 \times 10^{-3} \AA^{-2}$, for the three samples respectively. Small and positive value of $R$ for all samples show that electron density gradient at phase boundary is finite, suggesting that samples belong to a non-ideal two phase system.

Values of $R$ obtained by eq 5 by using five points forward difference method were found out to be $3.515 \times 10^{-3} \AA^{-2}, 2.323 \times 10^{-3} \AA^{-2}$, and $3.760 \times 10^{-3}$ 

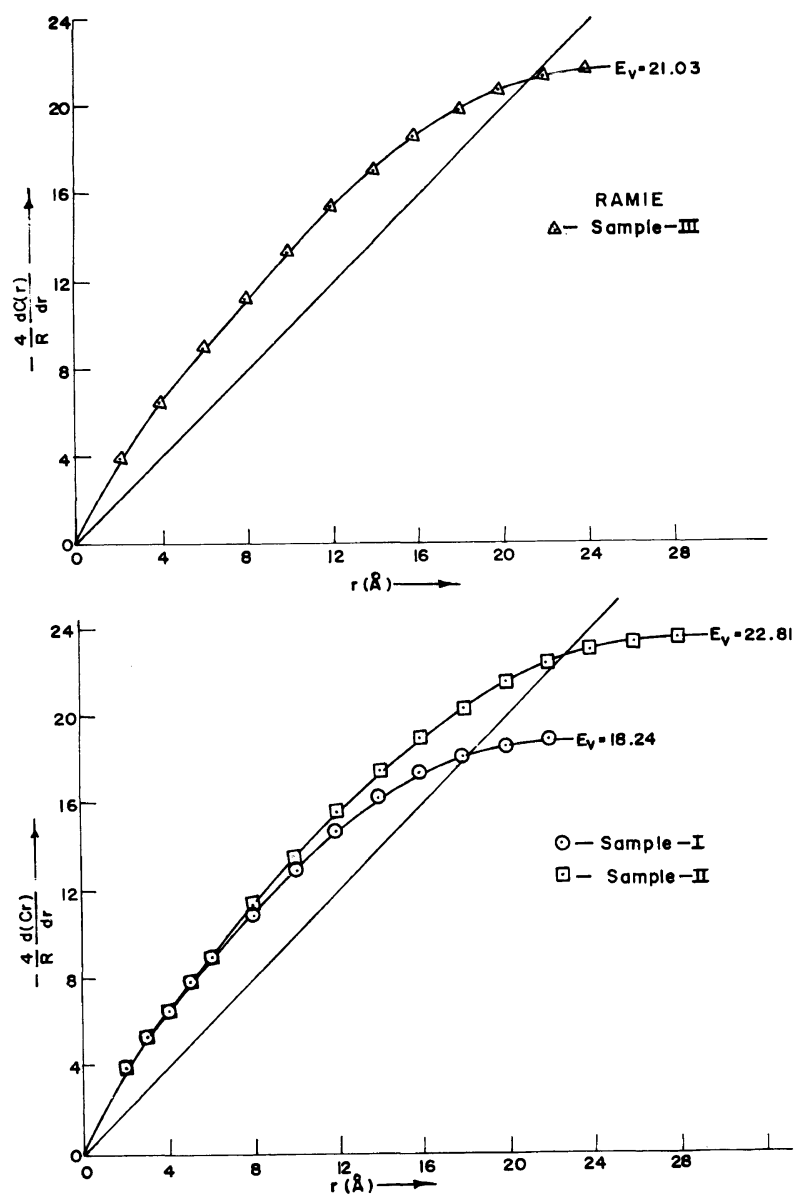

Figure 5. Curves of $-(4 / R)[\mathrm{d} c(r) / \mathrm{d} r]$ is. $r$.

Table I.

\begin{tabular}{cccc}
\hline Parameters & Sample-I & Sample-II & Sample-III \\
\hline$D / \AA$ & 219 & 265 & 373 \\
$E_{\mathrm{V}} / \AA$ & 18.246 & 22.829 & 21.030 \\
$E_{\mathrm{R}} / \AA$ & 18.131 & 19.755 & 19.029 \\
$S / V / \AA^{-1}$ & $9.132 \times 10^{-3}$ & $7.547 \times 10^{-3}$ & $5.362 \times 10^{-3}$ \\
$\phi_{1} / \%$ & 81.40 & 81.54 & 91.52 \\
$\phi_{2} / \%$ & 18.60 & 18.46 & 8.48 \\
$2 E_{\mathrm{V}} / D / \%$ & 16.66 & 17.22 & 11.28 \\
$T_{\mathrm{l}} / \AA$ & 356.00 & 432.14 & 682.73 \\
$T_{2} / \AA$ & 81.00 & 97.86 & 63.27 \\
$T_{\mathrm{r}} / \AA$ & 66.31 & 79.79 & 57.90 \\
$I_{\mathrm{c}} / \AA$ & 11.67 & 112.17 & 81.99 \\
$\sigma$ & 0.050 & 0.054 & 0.063 \\
$\gamma$ & 0.86 & 0.87 & 0.79 \\
& & & \\
\hline
\end{tabular}

$\AA^{-2}$ which is in agreement ot the values determined in first method, suggesting that samples under investigation are isotropic. The symmetric intensity pattern in Figure 2 also indicates the isotropic nature of the samples. The difference of intensity corresponding to the two types of mounting of samples is observed due to the difference of thickness of the samples. ${ }^{24}$

The three dimensional and one dimensional correlation function for all the samples were computed for various values of $r$ and $y$ respectively and are shown in Figures 3 and $4, E$ the width of transition layer is obtained form the plot of $(4 / R)[\mathrm{d} C(r) / \mathrm{d} r]$ Versus $r$, according to Vonk (Figure 5).

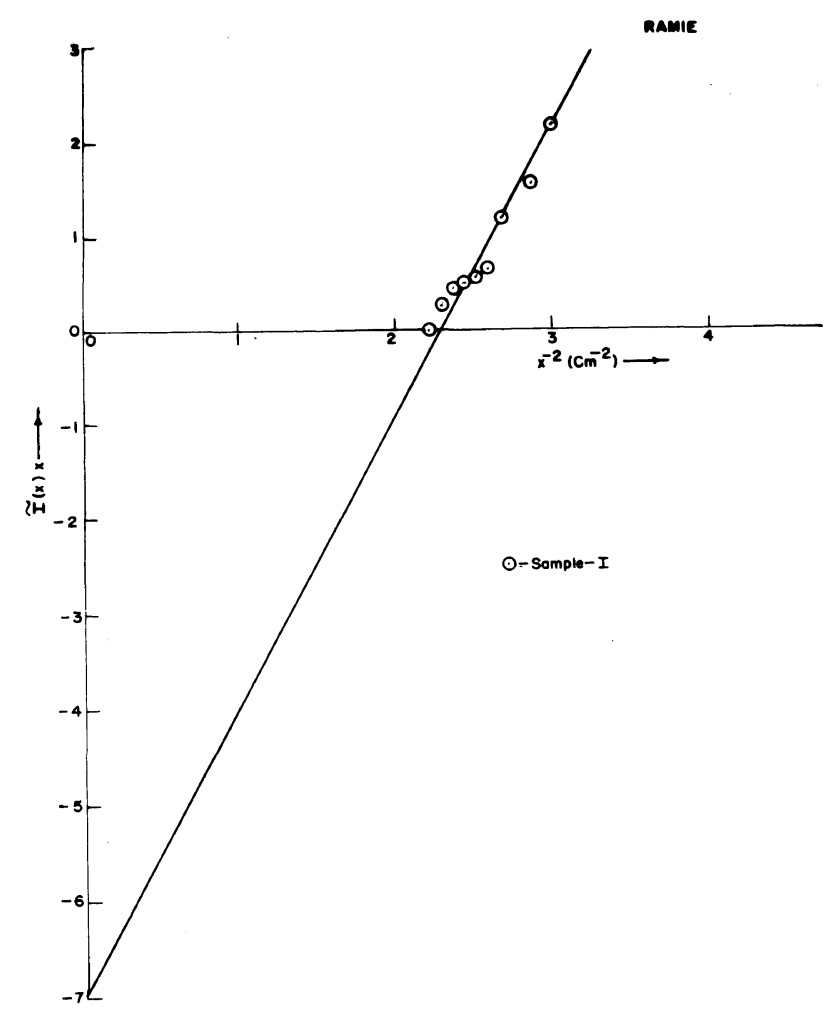

Figure 6a. Ruland plot of $I(x) x$ vs. $x^{-2}$.

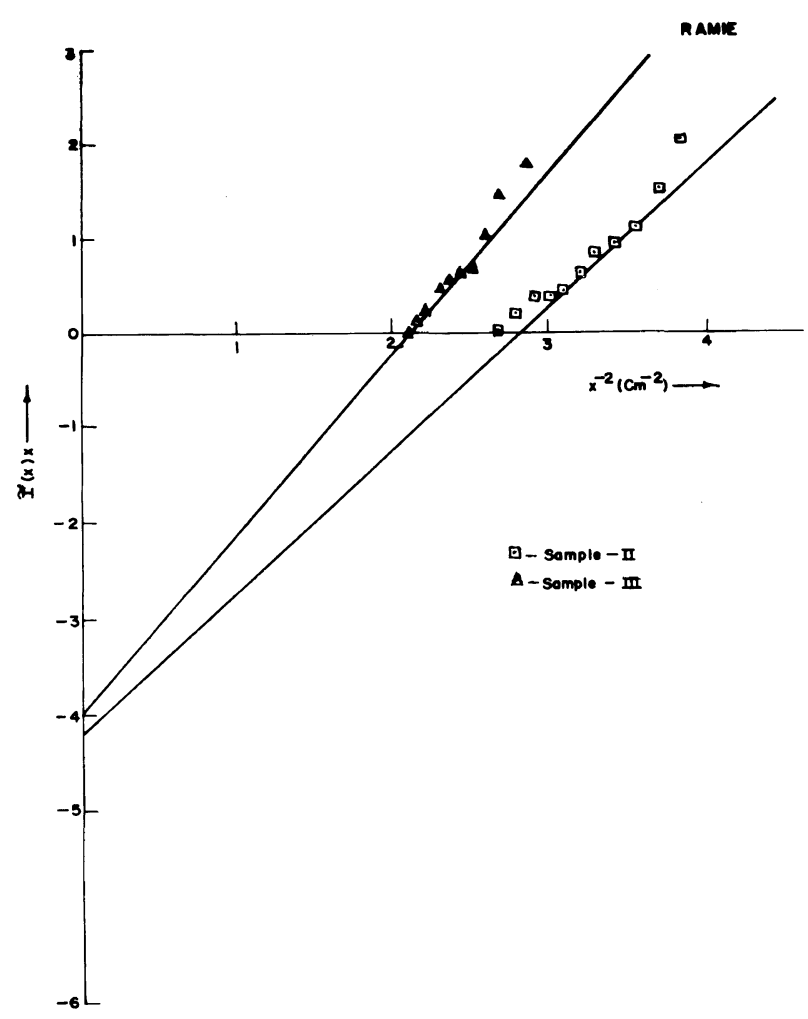

Figure 6b. Ruland plot of $I(x) x \vee s . x^{-2}$.

The value of $D$, the average periodicity transverse to layer were obtained as the position of first subsidiary maximum in one-dimensional correlation function Figure 4 . The values of $S / V$, the specific inner surface, $\phi_{1}$, the volume fraction of matter, $\phi_{2}$, the volume fraction of void, transversal lengths $\bar{l}_{1}$ (matter), $\bar{l}_{2}$ (void), $\bar{l}_{\mathrm{r}}$, range of inhomogeneties and ' $l_{c}$,' the length of coherence were 


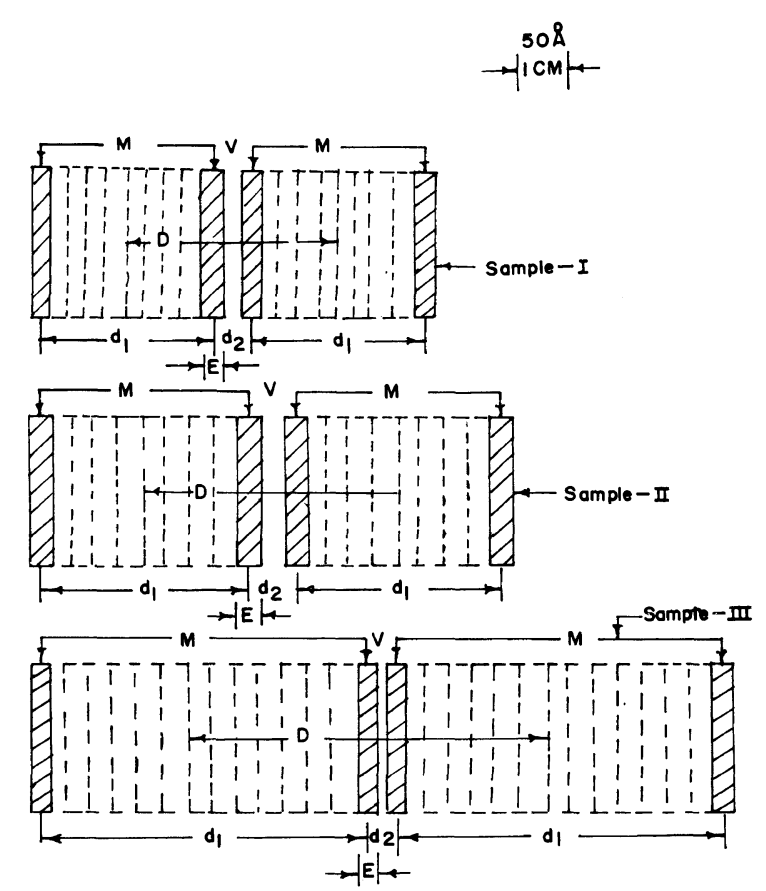

Figure 7. Lamellar models (drawn to scale), $M$, matter and $V$, void. $D$, periodicity transverse to layer; $E$, width of transition layer.

calculated, ' $d_{1}$ ' and ' $d_{2}$ ' the number average of thickness in two regions were also calculated. In Table I all the main SAXS parameters are shown. Lameller models for three samples are shown in Figure 7 . The broken lines in Figure 7 indicates crystalline region bounded by amorphous regions. Ruland plots $\tilde{I}(x) x$ versus $x^{-2}$ of the three samples are shown in Figures $6 \mathrm{a}$ and $6 \mathrm{~b}$ which gives straight lines. Slopes for samples are 3.046, 1.53, 1.961 , and $y$-intercepts are $-6.936,-4.141,-4.922$, for sample, I, II, and III, respectively and width of transition layer $E_{\mathrm{R}}$ by Ruland method was determined.

Standard deviation of intensities $\sigma$ was calculated at tail region of SAXS curve of samples and the values are well within the permissible limit. ${ }^{21}$ The regression coefficients $\gamma$ for all samples show that the data collected are fairly accurate. ${ }^{23}$

\section{CONCLUSION}

The values of $C(r)$ show damped oscillatory behavior at large values of $r^{16}$ for non-ideal two phase system and the trend is in evident for all the samples. Negative intercepts of Ruland plot confirms the non-ideal two phase nature. The values of width of transition layer, calculated following the methods of Vonk $\left(E_{\mathrm{V}}\right)$ and Ruland $\left(E_{\mathrm{R}}\right)$ are nearly equal confirm the correctness of data collection and method of analysis. The decrease in the value of specific inner surface $(S / V)$ after treatment with alkali shows that the fibre swells ${ }^{1}$ and it may be due to swelling to macromolecules of the fibre resulting in increase in value of $D$. Further our analysis shows that the macromolecules break (degrade) at higher $\mathrm{pH}$ value of sodium hydroxide solution, i.e., at $\mathrm{pH} 13$. Work on this line is in progress subjecting the sample to different physical and chemical conditions.

Acknowledgment. We are thankful to prof. A. K. Mohanty, Principal, Regional Engineering College, Rourkela for having granted permission to use the Computer and other facilities of Physics Department. We are also thankful to U.G.C. for granting Teachers fellowship to one of the authors J. Shukla to carry out the work.

\section{REFERENCES}

1. K. P. Sao, M. D. Mathew, and P. K. Ray, Text. Res. J., 407 (1987).

2. L. Cheek and L. Russel, Text. Res. J., 478 (1989).

3. T. Misra, D. K. Bisoi, T. Patel, K. C. Patra, and A. Patel, Polym. J., 20, 739 (1988).

4. T. Misra, Md. N. Khan, T. Patel, and N. V. Bhatt, J. Phys. D., Appl. Phys., 24, 331 (1991).

5. G. Porod, Kolloid. Z., 124, 83 (1951).

6. G. Porod, Kolloid. Z., 125, 51 (1952).

7. C. G. Vonk, J. Appl. Cryst., 6, 81 (1973).

8. N. B. Poul and S. K. Bhattacharya, J. Text. Inst., 12, 512 (1979)

9. S. C. Roy, Text. Res. J., 30, 451 (1966).

10. G. Porod, Kolloid. Z. Z. Polym., 133, 16 (1933).

11. T. Rotho and N. C. Sahu, Kolloid. Z. Z. Polym., 236, 43 (1970).

12. O. Glatter and O. Kratky, "Small Angle X-Ray Scattering," Academic press, New York, N. Y., 1981, p 435.

13. R. W. Hendrecks, J. Appl. Cryst., 3, 348 (1970).

14. W. Ruland, J. Appl. Cryst., 4, 70 (1971).

15. J. Mering and D. Tchoubar, J. Appl. Cryst., 1, 153 (1965).

16. T. Misra, K. C. Patra, and T. Patel, Colloid. Polym. Sci., 262, 611 (1984).

17. G. Kortleve and C. G. Vonk, Kolloid. Z. Z. Polym., 225, 124 (1971).

18. P. Mittelbach and G. Porod, Kolloid. Z. Z. Polym., 202, 40 (1965).

19. W. Rulland, Colloid Polym., 255, 417 (1977).

20. C. G. Vonk, J. Appl. Cryst., 8, 340 (1975).

21. C. G. Vonk, J. Appl. Cryst., 4, 340 (1971).

22. G. Konard and N. C. Zachmann, Kolloid. Z. Z. Polym., 247, 851 (1971).

23. J. Topping, "Errors of Observation and Their Treatment," Chapman and Hall, London, 1922, p 82 and p 104.

24. T. Misra, P. Panda, T. Patel, D. K. Bisoyi, and D. K. Panda, Jpn. J. Appl-Phys., 32, 4165 (1993). 